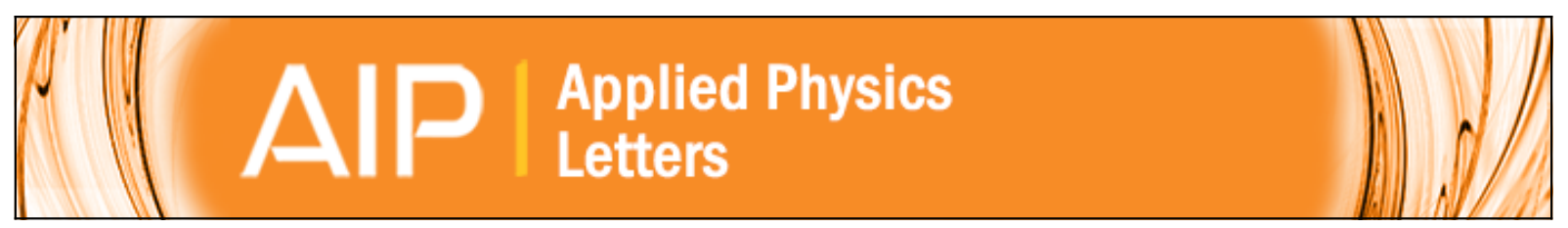

\title{
Highly stable superhydrophobic surfaces under flow conditions
}

Moonchan Lee, Changyong Yim, and Sangmin Jeon

Citation: Applied Physics Letters 106, 011605 (2015); doi: 10.1063/1.4905653

View online: http://dx.doi.org/10.1063/1.4905653

View Table of Contents: http://scitation.aip.org/content/aip/journal/apl/106/1?ver=pdfcov

Published by the AIP Publishing

\section{Articles you may be interested in}

Biomimetic hierarchical $\mathrm{ZnO}$ structures with superhydrophobic property

AIP Conf. Proc. 1502, 436 (2012); 10.1063/1.4769162

Predicting shape and stability of air-water interface on superhydrophobic surfaces comprised of pores with arbitrary shapes and depths

Appl. Phys. Lett. 100, 013104 (2012); 10.1063/1.3673619

Facile creation of bio-inspired superhydrophobic Ce-based metallic glass surfaces

Appl. Phys. Lett. 99, 261905 (2011); 10.1063/1.3672036

Reversible ultraviolet light-manipulated superhydrophobic-to-superhydrophilic transition on a tubular SiC nanostructure film

Appl. Phys. Lett. 97, 183112 (2010); 10.1063/1.3510472

Nanodesign of superhydrophobic surfaces

J. Appl. Phys. 106, 024305 (2009); 10.1063/1.3176484

LIVE DEMO

The Basics of COMSOL in 18 Minutes

จ С๐MSOL

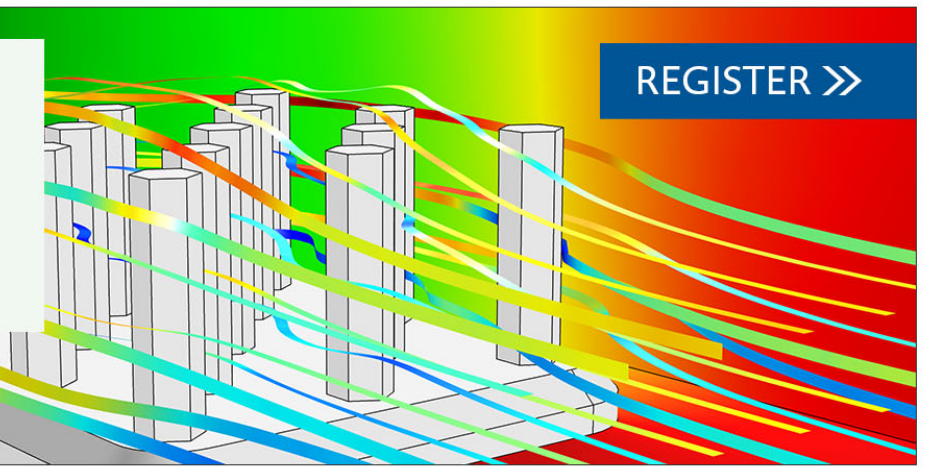




\title{
Highly stable superhydrophobic surfaces under flow conditions
}

\author{
Moonchan Lee, Changyong Yim, and Sangmin Jeon ${ }^{\text {a) }}$ \\ Department of Chemical Engineering, Pohang University of Science and Technology (POSTECH), \\ San 31 Namgu Hyojadong, Pohang 790-784, South Korea
}

(Received 16 October 2014; accepted 26 December 2014; published online 7 January 2015)

\begin{abstract}
We synthesized hydrophobic anodic aluminum oxide nanostructures with pore diameters of 35,50 , 65 , and $80 \mathrm{~nm}$ directly on quartz crystal microresonators, and the stability of the resulting superhydrophobicity was investigated under flow conditions by measuring changes in the resonance frequency and dissipation factor. When the quartz substrates were immersed in water, their hydrophobic surfaces did not wet due to the presence of an air interlayer. The air interlayer was gradually replaced by water over time, which caused decreases in the resonance frequency (i.e., increases in mass) and increases in the dissipation factor (i.e., increases in viscous damping). Although the water contact angles of the nanostructures increased with increasing pore size, the stability of their superhydrophobicity increased with decreasing pore size under both static conditions (without flow) and dynamic conditions (with flow); this increase can be attributed to an increase in the solid surface area that interacts with the air layer above the nanopores as the pore size decreases. Further, the effects of increasing the flow rate on the stability of the superhydrophobicity were quantitatively determined. (C) 2015 AIP Publishing LLC.

[http://dx.doi.org/10.1063/1.4905653]
\end{abstract}

Superhydrophobic surfaces are non-wetting, have large water contact angles that exceed $150^{\circ}$, and are easily prepared. ${ }^{1-3}$ Superhydrophobic surfaces have attracted much attention in recent decades because of their potential uses in a wide range of applications such as waterproof fabrics, humidity-repellent electronic devices, and oil-water separation filters. ${ }^{4-7}$ When a superhydrophobic surface is immersed in water, a thin layer of air between the water and the surface suppresses the direct contact of water with the surface. ${ }^{8-10}$ Superhydrophobic surfaces are potentially invaluable for ships because their non-wetting properties reduce hydrodynamic drag, thereby increasing fuel efficiency. ${ }^{11,12}$ In order for superhydrophobic coatings to be used in this way, they must have long-term stability in water. Most studies, however, have focused on the fabrication of superhydrophobic surfaces and only a few have reported their stability in water. ${ }^{13-16}$

Lee and Yong used a video camera to investigate the influence of hydrostatic pressure on the stability of $\mathrm{W}_{18} \mathrm{O}_{49}$ nanowire-grown superhydrophobic surfaces with various surface chemistries. ${ }^{15}$ The total reflection of white light between the water layer and the air pockets produced images of silvery surfaces, and the numbers of white and black pixels was converted to the surface areas of the nonwetted and wetted regions of the surface, respectively. They found that surfaces with large water contact angles are more resistant to surface wetting during immersion in water than surfaces with small contact angles. Although this result seems to apply to all superhydrophobic surfaces, an examination of its validity is required for various types of superhydrophobic surfaces, especially for surfaces with various air pocket shapes.

\footnotetext{
a) Author to whom correspondence should be addressed. E-mail: jeons@postech.ac.kr
}

In the present study, we synthesized anodic aluminum oxide (AAO)-based superhydrophobic nanostructures with sealed air pockets (i.e., the air pockets inside the AAO nanopores are isolated from each other) and $\mathrm{ZnO}$ nanorod-based superhydrophobic surfaces with open air pockets (i.e., the air pockets embedded between the $\mathrm{ZnO}$ nanorods are connected). Each of the nanostructures was grown directly on a quartz crystal microresonator (QCM) and the stability of its superhydrophobicity in water was evaluated in situ by analyzing the changes in the QCM's resonance frequency and dissipation factor. The acoustic wave generated by a vibrating quartz crystal decays rapidly with the distance from the crystal surface, so the decay length is just $\sim 250 \mathrm{~nm}$ in water at room temperature, which means that the QCM is sensitive to changes in mass or viscous damping only near the surface (i.e., surface wetting). ${ }^{17-19}$ We utilized this advantage of QCMs to investigate the influence of water flow rates on the stability of superhydrophobicity.

An aluminum layer was vacuum-deposited onto a quartz substrate and a two-step anodization process was carried out to synthesize a nanoporous AAO structure. ${ }^{16,20}$ The pore diameter, pore-to-pore distance, and height of the resulting AAO nanostructure were $35 \mathrm{~nm}, 100 \mathrm{~nm}$, and $1.2 \mu \mathrm{m}$, respectively. Pore widening was conducted for various durations and AAO nanostructures with various pore diameters were produced, as shown in the scanning electron microscopy (SEM) images in Figures 1(a)-1(d): $35 \mathrm{~nm}$ (AAO35), $50 \mathrm{~nm}$ (AAO50), $65 \mathrm{~nm}$ (AAO65), and $80 \mathrm{~nm}$ (AAO80). Figures 1(e) and 1(f) show a cross-sectional SEM image of AAO35 and an optical microscopy image of an AAO-grown quartz crystal substrate, respectively. The resonance frequencies and $Q$-factors of the AAO-grown quartz crystals were determined in air. Figure $1(\mathrm{~g})$ shows that the resonance frequency increases with pore size due to the decreases in mass loading, whereas the $Q$-factor decreases with pore size due to the 

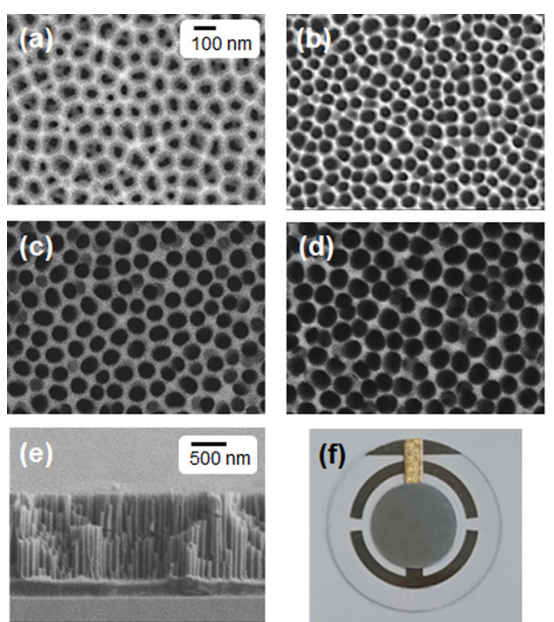

(g)

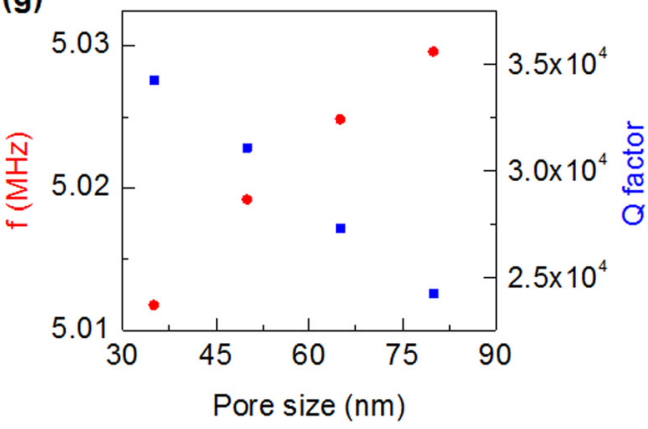

FIG. 1. SEM images of the AAO nanostructures with various pore diameters: (a) AAO35, (b) AAO50, (c) AAO65, and (d) AAO80. (e) Cross-sectional SEM image of AAO35. (f) Optical microscopy image of a quartz crystal microresonator with an AAO nanostructure. The diameter of the nanostructure pattern on the quartz crystal substrate is $1.27 \mathrm{~cm}$. (g) Variations with pore size in the resonance frequency (red circles) and $Q$-factor (blue squares) of the AAO-grown quartz crystal substrates. increases in the acoustic impedance (i.e., the increases in the void fraction) of the AAO layers.

To investigate the dependence of the surface wetting of the AAO structures on pore size, the hydrophilic AAOgrown quartz crystals (without hydrophobic modification) were placed inside a flow cell and the variations with pore size in their resonance frequencies and dissipation factors ( $D=1 / Q$, the inverse of the $Q$-factor) were measured as the surrounding medium changed from air to water. The immersion depth of the quartz crystal was controlled at $5 \mathrm{~mm}$ inside the flow cell to maintain constant hydrostatic pressure. Figure 2(a) shows the time-dependent changes in the resonance frequencies of the hydrophilic AAO-grown quartz substrates. Large changes in their resonance frequencies occurred immediately upon immersion in water. However, none of the resonance frequencies changed further after these initial changes, indicating that the hydrophilic AAO surfaces were immediately wetted upon immersion. Note that the change in frequency upon immersion in water increased with the pore size $(1701,2609,3601$, and $4596 \mathrm{~Hz}$ for AAO35, AAO50, AAO65, and AAO80, respectively), whereas the changes in the dissipation factor were nearly identical for all pore sizes. The changes in resonance frequency are affected by the amount of water loaded above and inside the nanopores. The water trapped inside the nanopores vibrates inphase with the quartz crystal and behaves like an ideal mass loading. As a result, the change in the resonance frequency
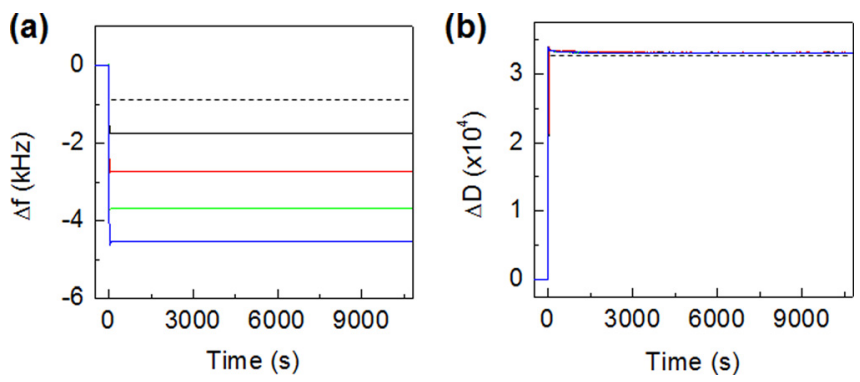

FIG. 2. The variations in (a) the resonance frequencies and (b) the dissipation factors of various hydrophilic quartz crystal substrates upon submersion in water: AAO35 (black), AAO50 (red), AAO65 (green), and AAO80 (blue). The dotted lines are the results for the plain Al-coated quartz crystal. increases as the pore size increases. In contrast, the water above the nanopores is viscously coupled with the surface and vibrates out-of-phase with the surface. The increase in viscous damping due to the water above the nanopores affects the dissipation factor as well as the resonance frequency.

To calculate the mass of water trapped inside the nanopores of each AAO surface, we determined the effects of water loaded above the nanopores on the resonance frequency. For this purpose, a plain Al-coated quartz substrate (i.e., an Al-deposited quartz substrate without nanopores) was immersed in water, and the changes in its resonance frequency $\left(\Delta f_{A l}: 856.3 \mathrm{~Hz}\right)$ and dissipation factor $\left(\Delta D_{A l}: \sim 3.3\right.$ $\times 10^{-4}$ ) were measured. Then, the mass of water trapped inside the nanopores of each AAO surface can be calculated by using the Sauerbrey equation ${ }^{21}$

$$
\Delta m=-\frac{A \sqrt{\rho_{q} \mu_{q}}}{2 f_{0}^{2}} \Delta f,
$$

where $\Delta f$ is the difference between the frequency change obtained for each AAO surface and $\Delta f_{A l}, A\left(\mathrm{~cm}^{2}\right)$ is the active area of the quartz crystal, $f_{0}$ is the resonance frequency of the unloaded quartz crystal, $\rho_{\mathrm{q}}\left(2.648 \mathrm{~g} / \mathrm{cm}^{3}\right)$ is the density of quartz, and $\mu_{\mathrm{q}}\left(2.947 \times 10^{11} \mathrm{~g} /\left(\mathrm{cm} \mathrm{s}^{2}\right)\right)$ is the shear modulus of quartz. The masses of water trapped inside the nanopores of AAO35, AAO50, AAO65, and AAO80 were calculated to be $18.13,37.64,58.92$, and $80.29 \mu \mathrm{g}$, respectively.

Figure 2(b) shows the changes with time in the dissipation factors of the hydrophilic AAO-grown quartz substrates with time. As for the changes in the resonance frequencies, large changes in the dissipation factors were evident immediately upon immersion in water and no further changes occurred after the initial changes, which confirms that the hydrophilic AAO surfaces were immediately wetted upon immersion. The dissipation factors of the AAO-grown quartz substrates were slightly larger than that of the plain Alcoated quartz substrate due to their higher surface roughness. However, they exhibited nearly identical changes in their dissipation factors for all pore sizes, which indicate that the differences between the surface roughnesses of the AAO surfaces were negligible. Note that a higher dissipation factor 
implies that the quartz surface experiences greater resistance during vibration in water.

The surfaces of the AAO nanostructures were chemically modified to render them hydrophobic by incubating them in $10 \mathrm{mM}$ octadecyltrichlorosilane (OTS) for $3 \mathrm{~h}$. The water contact angles were measured at eight different locations on each substrate and found to be $138.6 \pm 0.9^{\circ}$, $144.0 \pm 0.6^{\circ}, 149.7 \pm 0.3^{\circ}$, and $157.1 \pm 0.7^{\circ}$ for $\mathrm{AAO} 35$, AAO50, AAO65, and AAO80, respectively (see Figure S1 in the supplementary material). ${ }^{22}$ The increases in the contact angle with pore size can be attributed to decreases in the contact line between water and the nanostructure surfaces. ${ }^{23-25}$ Figure 3(a) shows the variations with time in the resonance frequencies of the hydrophobic AAO-grown quartz crystals upon immersion in water. These variations are quite different from those of the hydrophilic AAO nanostructures, in two significant ways. First, the initial changes in the resonance frequencies $\left(\Delta f_{0}: 810-850 \mathrm{~Hz}\right)$ of the hydrophobic AAO nanostructures upon water loading are small compared to those of the hydrophilic AAO nanostructures $(1700-4600 \mathrm{~Hz}$ in Figure 2(a)). These changes are even smaller than the change in the resonance frequency of the plain Al-coated quartz crystal upon immersion in water $(856.3 \mathrm{~Hz})$. This result indicates not only that water did not fill the hydrophobic nanopores but also that the surfaces of the hydrophobic or superhydrophobic AAO nanostructures were not completely wetted by immersion. This incomplete wetting indicates that a thin air interlayer was present between each hydrophobic AAO nanostructure and water.

The second significant difference is that there are gradual decreases in the resonance frequencies after the initial instant changes upon immersion in water. The inset in Figure 3(a) shows the changes in the resonance frequencies during surface wetting more clearly. After $12 \mathrm{~h}$ of immersion in water, the masses of water that replaced the air layers on AAO35, AAO50, AAO65, and AAO80 were calculated to be $0.42,0.88,1.55$, and $2.33 \mu \mathrm{g}$, respectively; these values are much smaller than the masses of water required to fill each nanopore fully $(<3 \%)$. Therefore, these gradual frequency changes are associated with the gradual wetting of the top surface caused by the replacement of the air interlayer with water rather than with the permeation of water into the nanopores. Note that the wetting rates
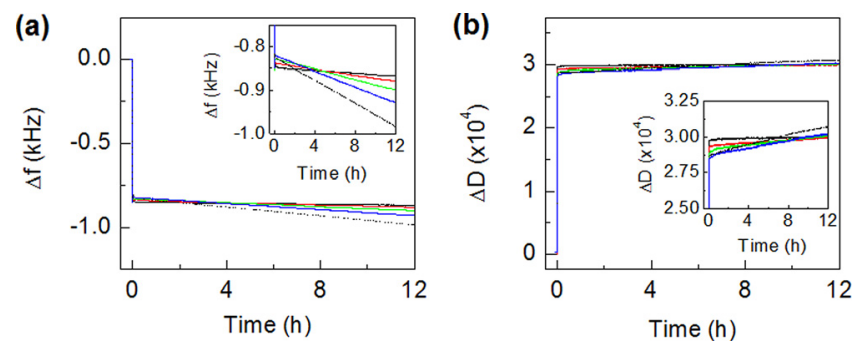

FIG. 3. The variations in (a) the resonance frequencies and (b) the dissipation factors of various hydrophobic quartz crystal substrates upon submersion in water: AAO35 (black), AAO50 (red), AAO65 (green), and AAO80 (blue). The dotted lines are the results for the $\mathrm{ZnO}$ nanorod-grown quartz crystal substrate. The insets show plots on modified axis scales of the variations in the respective signals. decreased in the order AAO80 $>$ AAO65 $>$ AAO50 $>$ $\mathrm{AAO} 35$; this trend is the opposite of that found for the water contact angles and of that found by Lee and Yong, ${ }^{15}$ who reported that superhydrophobic $\mathrm{W}_{18} \mathrm{O}_{49}$ nanowires with larger water contact angles exhibited greater underwater stability. These results suggest that the initial degree of surface wetting is determined by the water contact angle and that the rate of surface wetting after immersion is determined by surface morphology.

To further investigate the effects of surface morphology on the stability of the nanostructures' superhydrophobicity, a superhydrophobic $\mathrm{ZnO}$ nanorod structure was prepared on a quartz crystal substrate (see Figure S2 in the supplementary material). ${ }^{22}$ In contrast to the AAO nanostructures, which contain sealed air pockets, open air pockets are embedded between the $\mathrm{ZnO}$ nanorods. ${ }^{23,26,27}$ The surface of the $\mathrm{ZnO}$ nanorods with a height of $1.2 \mu \mathrm{m}$ was rendered hydrophobic by treatment with $10 \mathrm{mM}$ octadecanethiol (ODT) and the water contact angle was measured to be $154.3 \pm 0.5^{\circ}$. Figure 3(a) shows that the rate of surface wetting of the hydrophobic $\mathrm{ZnO}$ nanorods was faster than those of the hydrophobic AAO surfaces, which indicates that the sealed air pockets inside the AAO nanopores were more stable than the open air pockets between the $\mathrm{ZnO}$ nanorods. As for the resonance frequency changes, the dissipation factor increased instantly upon immersion in water and then gradually increased over time, as shown in Figure 3(b). The inset in Figure 3(b) shows that wetting was faster for hydrophobic AAO surfaces with larger pore sizes and that the hydrophobic AAO surfaces were more resistant to surface wetting than the hydrophobic $\mathrm{ZnO}$ surface.

In addition to the static stability of the superhydrophobicity of the nanostructures, its dynamic stability at various external flow rates was examined. Hydrophobic AAO35, AAO80, and $\mathrm{ZnO}$ nanorod-grown quartz crystal substrates were immersed in water at various flow rates $(0,10,25$, and $50 \mu \mathrm{l} / \mathrm{min}$ ) and their resonance frequencies and dissipation factors were monitored (see Figure S3 in the supplementary material). ${ }^{22}$ Figures 4(a) and 4(b) show the variations in the derivative of the resonance frequencies $\left(R_{\mathrm{f}}\right)$ and the dissipation factors $\left(R_{\mathrm{D}}\right)$ as a function of flow rates. Both figures show that the surface wetting rate increased with the flow rate for all three superhydrophobic nanostructures and that the surface wetting of the $\mathrm{ZnO}$ nanorods was the most severely affected by the external flow due to its open air
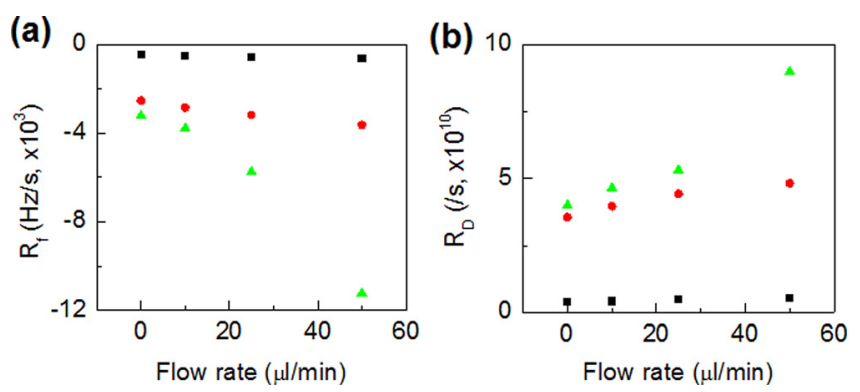

FIG. 4. Variations with the flow rate in the surface wetting of various hydrophobic nanostructures: (a) resonance frequencies and (b) dissipation factors (black squares: AAO35, red circles: AAO80, and green triangles: $\mathrm{ZnO}$ nanorods). 
pockets. This result confirms that the air clusters embedded between the hydrophobic $\mathrm{ZnO}$ nanorods are vulnerable to the surrounding flow. In contrast to the poor stability of the hydrophobicity of the $\mathrm{ZnO}$ nanorods, the hydrophobicities of the AAO structures were not strongly affected by the external flows because the air clusters are stably embedded inside the nanopores. The AAO nanostructure with the largest pores (AAO80) was more sensitive to external water flow than the structure with small pores (AAO35) because the solid surface area that interacts with the air layers above the nanopores was larger for AAO35 than for AAO80. After $12 \mathrm{~h}$ of immersion in a $50 \mu \mathrm{l} / \mathrm{min}$ flow, the masses of water that had replaced the air interlayers on the AAO35 and AAO80 surfaces and on the $\mathrm{ZnO}$ nanorods were calculated to be $0.6,3.6$, and $11.4 \mu \mathrm{g}$, respectively; these masses were $1.42,1.54$, and 3.50 times greater than the replaced mass of water without any flow for $12 \mathrm{~h}$, respectively.

In summary, we synthesized various hydrophobic and superhydrophobic nanostructures directly on quartz crystal microresonators and investigated the effects of surface geometry on the stabilities of their superhydrophobicity under external water flows. The nanostructures synthesized in this study include $\mathrm{ZnO}$ nanorods and $\mathrm{AAO}$ nanostructures with four different pore diameters (AAO35, AAO50, AAO65, and AAO80). The air clusters embedded in nanostructures suppress the direct contact of water with the surface and maintain the non-wetting properties. The hydrophobic AAO nanostructures with sealed air pockets generally retained their embedded air clusters longer than the $\mathrm{ZnO}$ nanorods with open air pockets. Of the AAO nanostructures, AAO35 exhibited the best underwater stability in both static (without flow) and dynamic (with flow) conditions even though it had the smallest contact angle. These results are attributed to the air clusters that are stably embedded inside the AAO nanostructures and the largest solid surface area to interact with air layers above the nanopores.
This research was supported by a grant (14CTAPC077604-01) funded by Ministry of Land, Infrastructure and Transport of Korean government.

${ }^{1}$ C. Dorrer and J. Rühe, Soft Matter 5, 51 (2009).

${ }^{2}$ Y. T. Cheng and D. E. Rodak, Appl. Phys. Lett. 86, 144101 (2005).

${ }^{3}$ J. Xi, L. Feng, and L. Jiang, Appl. Phys. Lett. 92, 053102 (2008).

${ }^{4}$ L. Feng, S. H. Li, Y. S. Li, H. J. Li, L. J. Zhang, J. Zhai, Y. L. Song, B. Q. Liu, L. Jiang, and D. B. Zhu, Adv. Mater. 14, 1857 (2002).

${ }^{5}$ I. P. Parkin and R. G. Palgrave, J. Mater. Chem. 15, 1689 (2005).

${ }^{6}$ S. Lee, W. Kim, and K. Yong, Adv. Mater. 23, 4398 (2011).

${ }^{7}$ B. Wang and Z. Guo, Appl. Phys. Lett. 103, 063704 (2013).

${ }^{8}$ R. Poetes, K. Holtzmann, K. Franze, and U. Steiner, Phys. Rev. Lett. 105, 166104 (2010).

${ }^{9}$ I. A. Larmour, S. E. J. Bell, and G. C. Saunders, Angew. Chem. Int. Ed. 46, 1710 (2007).

${ }^{10}$ N. J. Shirtcliffe, G. McHale, M. I. Newton, C. C. Perry, and F. B. Pyatt, Appl. Phys. Lett. 89, 104106 (2006).

${ }^{11} \mathrm{~B}$. Su, M. Li, and Q. Lu, Langmuir 26, 6048 (2010).

${ }^{12}$ H. Dong, M. Cheng, Y. Zhang, H. Wei, and F. Shi, J. Mater. Chem. A 1, 5886 (2013).

${ }^{13}$ G. McHale, M. I. Newton, and N. J. Shirtcliffe, Soft Matter 6, 714 (2010).

${ }^{14}$ C. Lee and C. J. Kim, Phys. Rev. Lett. 106, 014502 (2011).

${ }^{15}$ J. Lee and K. Yong, J. Mater. Chem. 22, 20250 (2012).

${ }^{16}$ M. Lee, C. Yim, and S. Jeon, Langmuir 30, 7931 (2014).

${ }^{17}$ I. Goubaidoulline, G. Vidrich, and D. Johannsmann, Anal. Chem. 77, 615 (2005).

${ }^{18}$ W. Ko, N. Jung, M. Lee, M. Yun, and S. Jeon, ACS Nano 7, 6685 (2013).

${ }^{19}$ H. Seo, J. Joo, W. Ko, N. Jung, and S. Jeon, Nanotechnology 21, 505502 (2010).

${ }^{20}$ M. Lee, D. Lee, N. Jung, M. Yun, C. Yim, and S. Jeon, Appl. Phys. Lett. 98, 013107 (2011).

${ }^{21}$ G. Z. Sauerbrey, Z. Phys. 155, 206 (1959).

${ }^{22}$ See supplementary material at http://dx.doi.org/10.1063/1.4905653 for time-dependent changes in resonance frequencies and dissipation factors of hydrophobic nanostructures under external flow rates.

${ }^{23}$ Y. Lai, X. Gao, H. Zhuang, J. Huang, C. Lin, and L. Jiang, Adv. Mater. 21, 3799 (2009).

${ }^{24}$ N. Tasaltin, D. Sanli, A. Jonáš, A. Kiraz, and C. Erkey, Nanoscale Res. Lett. 6, 487 (2011).

${ }^{25}$ W. Lee, B. G. Park, D. H. Kim, D. J. Ahn, Y. Park, S. H. Lee, and K. B. Lee, Langmuir 26, 1412 (2010).

${ }^{26}$ S. Wang and L. Jiang, Adv. Mater. 19, 3423 (2007).

${ }^{27}$ M. Jin, X. Feng, L. Feng, T. Sun, J. Zhai, T. Li, and L. Jiang, Adv. Mater. 17, 1977 (2005). 